\title{
Vertical Handoff Prediction based on Human Movement and Little's Law
}

\author{
Alap Kango \\ Pimpri Chinchwad \\ College Of Engineering, \\ Department of Computer \\ Engineering, \\ Savitribai Phule Pune University
}

\author{
Shivdatta Patil \\ Pimpri Chinchwad \\ College Of Engineering, \\ Department of Computer \\ Engineering, \\ Savitribai Phule Pune University
}

\author{
Tejas Ghanekar \\ Pimpri Chinchwad \\ College Of Engineering, \\ Department of Computer \\ Engineering, \\ Savitribai Phule Pune University
}

\author{
Tejas Dhawale \\ Pimpri Chinchwad \\ College Of Engineering, \\ Department of Computer Engineering, \\ Savitribai Phule Pune University
}

\author{
S.S. Sambare \\ Pimpri Chinchwad \\ College Of Engineering, \\ Department of Computer Engineering, \\ Savitribai Phule Pune University
}

\begin{abstract}
Mobility in communication is an important factor now-adays.The mobile telecommunication service providers are trying to achieve maximum mobility. In mobile network there are many factors affecting mobility. It is thus necessary to look after the bandwidth strength, RSS, load balancing and many other vital factors. This paper has explained a very important mechanism of mobile networks which is handoff and its various types. The prediction part and the various existing strategies come into play and have a role in deciding the activities based on user movement. It is immensely important to predict the user movement and keep the resources ready which will be helpful to achieve a seamless handoff. Such predictions can be useful to minimize the delay, call drops and have real life application in traffic management and disease spread prediction.
\end{abstract}

\section{Keywords}

RSS, load balancing, handoff, Quality of Service(QoS), Base Stations, mobility management, trilateration, Little's law, Human movement, mobile computing

\section{INTRODUCTION}

Today's wireless networking system should be comprised of essential features like seamless mobility and roaming. To attain mobility in wireless networks, handoff mechanism can be used. When a mobile terminal changes its point of access, there is a need of handoff and it is the process of switching the channel (frequency, time slot, spreading code, or combination of them) associated with the present connection while a call is in progress[2]. Multiple wireless networks provide a wide range of applications that are accessed by mobile users using multiple interfaces. Therefore, the structural design of the networks must be such that the related functions must work independent of the network technology. The most important job of the service providers is to deliver their network services in an efficient manner. Depending on the Quality of Service requirement of the application, the network selection criteria may vary. To accomplish this goal, the decision making strategy for a specific application must be very efficient [3].

\section{HANDOFF}

All The handoffs are basically classified into two main types based on their functioning.

\subsection{Horizontal Handoff (HHO)}

The Handoff that takes place between two base stations (BSs) of the same system is called as Horizontal handoff. It involves a terminal device which is used to change cells within the same type of network (e.g., within a CDMA network) for a maintained service continuity [4]. Its further classification is done as Link-layer handoff and Intra-system handoff. Horizontal handoff between two BS, under same Foreign Agent (FA) is known as Link-layer handoff[2]. Whereas in Intra-system handoff, the horizontal handoff occurs between two Base Stations that belong to two different FAs and both FAs belongs to the same system and hence to same Gateway Foreign Agent (GFA) [2].

\subsection{Vertical Handoff (VHO)}

The process in which a node changes the type of connectivity it uses for accessing a supporting infrastructure, usually to support node mobility is known as Vertical Handoff [2]. Vertical handovers refers to the automatic switch from one technology to another to maintain communication. A change in network between different types of networks (e.g., between $3 \mathrm{G}$ and $4 \mathrm{G}$ networks) which is completely transparent to the end user application, is allowed by the vertical handoff mechanism[2].

The three main phases of vertical handoff are system discovery, vertical handoff decision and VHO execution.

\subsubsection{System discovery}

The networks which can be used by the mobile terminal are determined by it. The supported data rates and Quality of Service (QoS) parameters may also be advertised by these networks. 


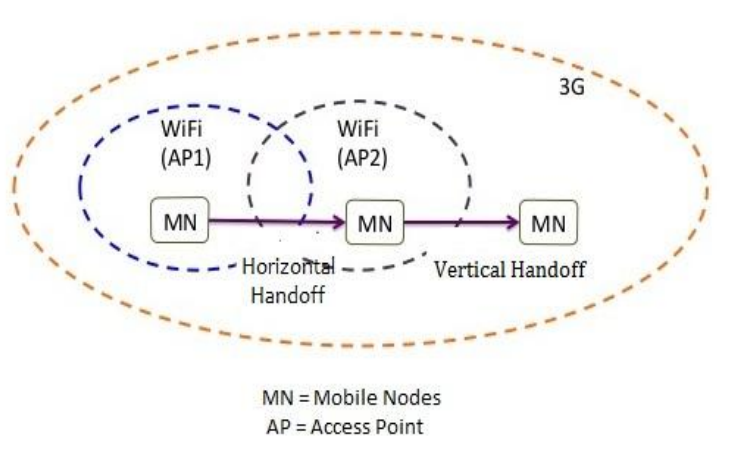

Figure 1: Horizontal and Vertical Handoff

\subsection{2 $\mathrm{VHO}$ decision}

If the connections should continue using the current network or be switched to another network is determined by the mobile terminal. Various parameters or metrics including the type of the application (e.g., conversational, streaming), minimum bandwidth and delay required by the application, access cost; transmit power, and the user's preferences are responsible for the decision that is to be made.

\subsubsection{VHO execution}

Re-routing of connections in mobile terminal from the existing network to the new network in a seamless manner is done in this phase. This phase includes the authentication, authorization, and transfer of a user's context information [5].

\section{REQUIREMENT FOR HANDOFF MECHANISM}

The classification of requirement for handoff mechanism is done into dynamic and non-dynamic requirements. The dynamic requirements include RSS, velocity, throughput, user preferences, handover latency, network load balancing as parameters and non-dynamic requirements include network cost, power consumption, network security and bandwidth as parameters [6].

\subsection{Bandwidth}

Bandwidth needs to be managed efficiently for achieving seamless handoff for Quality of service (QoS) in wireless environment. Bandwidth is generally known as the link capacity in a network. Lower call dropping and call blocking probabilities are ensured by higher offered bandwidth hence higher throughput can be obtained.

\subsection{Consumption of Power}

Energy efficiency is a major concern in wireless networks. Mobile switching or handoffs consume power. Frequent interface activation during handoffs can cause considerable battery drainage. Unnecessary interface activation can give rise to the issue of power saving and can also increase power consumption during network discovery. It is also important to incorporate power consumption factor during handoff decision.

\subsection{Latency of handoff}

During the process of Handover of calls between two BS (a frequent phenomenon) a delay can occur. This delay is known as handoff latency. A good handoff decision model should consider minimizing handoff latency. Service quality of many applications of mobile users is affected by handoff latencies.

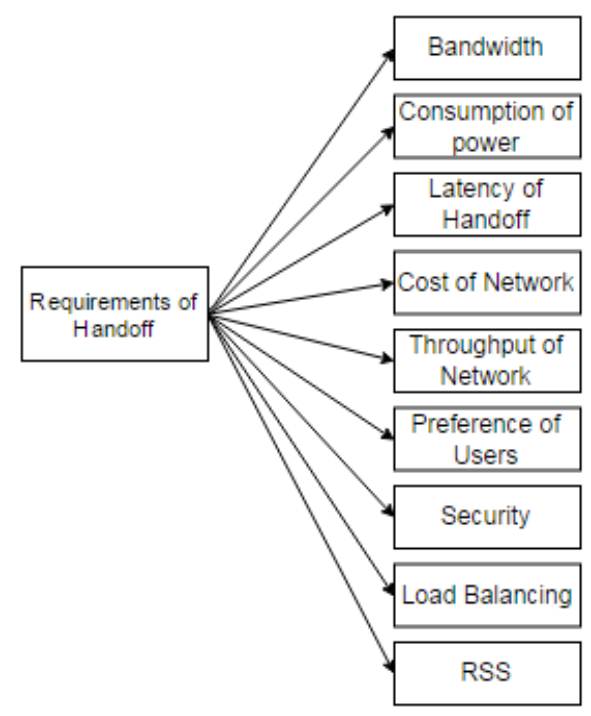

Figure 2: Requirements of Handoff.

\subsection{Cost of Network}

The cost is to be minimized during VHO in wireless networks handover. The cost function can be used to analyze the new arrival rates and handoff call arrival rates. Advantages on coverage and data rates can be combined by the respective next generation heterogeneous networks, offering a high Quality of Service (QoS) to mobile users. Therefore, network selection cost is important in handoff decisions.

\subsection{Throughput of Network}

Network throughput is the average data rate of successful data or message delivery over a specific communications link. It is measured in bits per second (bps). Maximum network throughput is equal to the TCP window size divided by the round-trip time (RTT) of communications data packets. It is one of the most important requirements to be considered for the VHO.

\subsection{Preference of users}

According to the preferences and network performance parameters, the users have more options for heterogeneous networks during handoff. The user preferences could be preferred networks, user application requirements (real time, non-real time), service types (Voice, data, video), QoS etc. User Preferences can also be considered for $\mathrm{VHO}$ in $4 \mathrm{G}$ wireless networks.

\subsection{Security}

Secure handoff is another important factor in wireless networks. The network security consists of the provisions and policies adopted by the network for preventing and monitoring unauthorized access, misuse, modification, and network-accessible resources. Highest levels of integrity, authentication, and confidentiality are essential security features that should be embedded in the handoff policies.

\subsection{Load Balancing}

Balancing of network load is another important task, to avoid deterioration in quality of services. Variations in the traffic loads among cells will reduce the traffic-carrying capacity. Network load must be paid attention and network load is to be considered during effective handoff to provide a high quality communication service for mobile subscribers and to enhance a high traffic-carrying capacity when there are variations in traffic 


\subsection{Received Signal strength (RSS)}

The wireless networks, performance depends on signal strength of mobile node (MN) and access point (AP).The total amount of network bandwidth available along that connection is determined by the wireless signal strength in each direction. RSS depicts the power present in a received signal. To maintain signal quality at receiver end it is necessary that a signal must be strong enough between BS and mobile unit. The RSS should maintain a specific threshold in a network during handoff.

\section{PREDICTION FOR HANDOFF}

The monitoring of the users movement can be achieved with the help of mobile telecom service so as to track the user movement. The mobile telecom network makes use mobility management mechanism so that the network can connect incoming calls to the users through Base Stations (BSs).In order to get users location BSs in the service area (SA) are grouped into location areas (LAs).[7]

Since mobility management mechanism provides the position information of a user at a low accuracy and thus it is not possible to apply it in location based applications that require precise procedure accuracy.

\subsection{Existing techniques for location mapping}

1) The "Observed Time Difference of Arrival (OTDOA)" method uses trilateration to find out the mobile user's position. So what's trilateration, in at least three concurrent downlink signals from different cells are measured by the mobile phone in this process. The time differences of the various signal arrivals are calculated in order to form hyperbolic curves. The intersection of these curves indicate the mobile user's position. Location accuracy provided by this method is within 50-150 meters. [1]

2) The "Assisted Global Positioning System (A-GPS)" method speeds up the calculation of GPS positioning by downloading GPS related information through the radio access network (RAN). This process of vital information gathering is faster than execution of normal GPS positioning. GPS modules are preinstalled in mobile phone and RAN both .This method has higher location accuracy within 5-15 meters. [1]

3) Another method is of "Uplink Time Difference of Arrival (U-TDOA)" method evolves from OTDOA, it utilizes uplink signals instead of downlink signals. Usually the uplink signal from the mobile user is measured in different cells. This method does not require any modification to the mobile phone as calculations are done in RAN. The location accuracy is 50150 meters. [1]

\section{LIMITATIONS OF EXISTING SYSTEMS}

The techniques mentioned above can effectively monitor the behaviors of specific mobile users at the cost of modifications to telecom network but the change required in the mobile telecom will be huge and won't generate the required large amount user statistics. In other words it can also be stated that these are not that useful to find precise or to the point exact probability of a user changing a cell or no. For this a location with such a method which can help us get the probability needed as an output is required. The output shall answer the question such as: "What is the probability $\mathrm{P}$ of A,B ; a person at location A will move to location B after T hours."

\subsection{Spread Model Prediction}

In [8] this paper, an approach to address the spread problem by only using the statistics to be taken from the standard mobile telecom switches such as mobile switching centers (MSCs), and serving general packet radio service support nodes (SGSNs) are cited.This approach does not need to identify individual users and, therefore, does not cause any customer privacy problem.

A mobile telecom SA covered by several BSs. In this figure, a cell of a BS is represented by a circle. The mobile user is represented by a vehicle moving around the cells. If a user in conversation moves from one cell to another, the call connection is bound to switch from the old cell to the new cell.

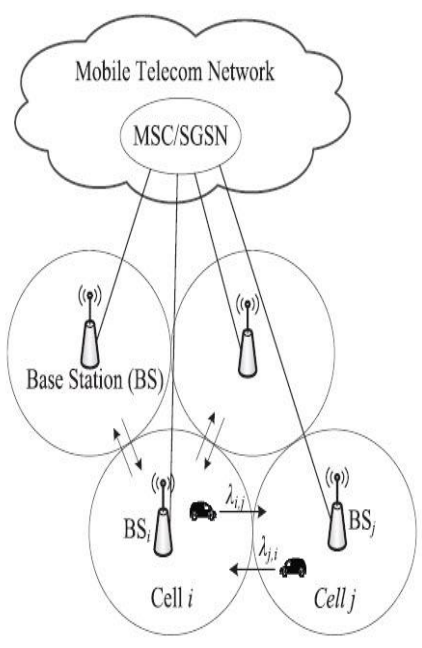

Figure 3: Mobile telecom network

This switching operation is called a handover. When a call arrives at a user or when he/she performs a handover and this activity is recorded at the MSC/SGSN. The mobile telecom network collects the statistics of the activities for every at interval typically ranging from 15 minutes to several hours.

The concept behind the prediction model is Little's Law, which says that the expected number $\mathrm{N}$ of users in a system is the arrival rate $\lambda$ of the users times the expected response time $\mathrm{R}$ that a user stays in the system; i.e.,

$$
\mathrm{N}=\lambda \mathrm{R}
$$

The average number ( )of users at cell $\mathrm{i}$ in timeslot $\Delta \tau$ is given by

$$
\operatorname{Ni}(\tau)=\rho i(\tau) / E[t c]
$$

Major four measures provided by telecom network are:

1) The expected call holding time

2) The numbers of handovers in and out of the cells

3) The number of new call arrivals of the cells

4) The voice/data traffic (in Erlang) cells. 


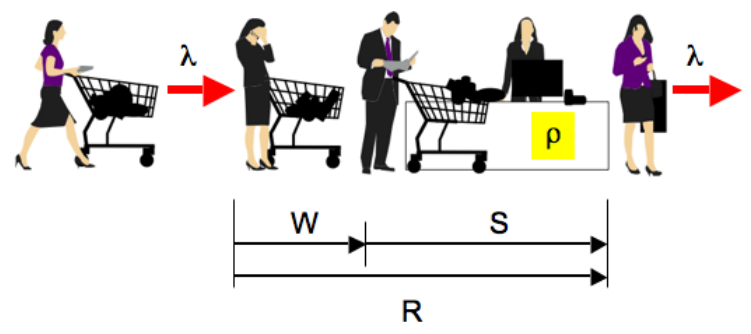

Figure 4. Little's Law

\section{CONCLUSION}

Based on Little's Law, this paper proposes a model to predict how people move from one location to another after a random interval of time. This information is very useful to investigate issues such as prediction of vehicle traffic and spread of contagious diseases. The standard statistics provided by a commercial mobile telecom network are used as inputs for the prediction model. The prediction model will generate a probability as an output which shall help in decision making, it will tell if the handoff will occur or not and it will help the system keep resources ready. Existing solutions fail to answer the question "What is the probability that a person at location A will move to location B after T hours?" with statistics of large samples of human movements. Instead of using dynamic data experiments will be carried on same amount of static data. This size of data shall be increased later that would help to achieve an algorithm which shall accept huge number of dynamic user statistics.

\section{ACKNOWLEDGMENT}

We would like to thank our project guide Prof.S.S.Sambare for his enormous co-operation and guidance. We have no words to express our gratitude for a person who whole heartedly supported the project and gave freely of his valuable time while making this project. The technical guidance provided by him was more than useful and made the project successful. We would also like to thank our Department of Computer Engineering, Pimpri Chinchwad College of Engineering.

\section{REFERENCES}

[1] Yi-Bing Lin, Fellow, IEEE, Chien-Chun Huang-Fu, and Nabil Alrajeh Predicting Human Movement Based on Telecoms Handoff in Mobile Networks IEEE Transactions on Mobile Computing, VOL. 12, NO. 6, June 2013.

[2] Arvind Kumar Singh, Pankaj Singh, Sandeep Singh, Akhilesh Kumar Singh "Handover in Advanced Wireless communication A Survey"IJCSET ,September 2011,Vol 1 , Issue 8, 510-515.

[3] A. Bhuvaneswari,"An Overview of Vertical Handoff Decision Making Algorithms", I. J. Computer Network and Information Security, 9, 55-62 August 2012 in MECS (http://www.mecs-press.org/).
[4] Abdoul-Aziz IssakaHassane, Li Renfa, and ZengFanzi Handover Decision Based on User Preferences in Heterogeneous Wireless Networks -College of Information Science and Engineering, Hunan University, China 2012

[5] B. R. Chandavarkar, G. Ram Mohan Reddy - Survey Paper: Mobility Management in Heterogeneous Wireless Networks\| Department of Information Technology National Institute of Technology Karnataka, Surathkal, Mangalore, India 2011.

[6] Mandeep Kaur Gondara1 and Dr. Sanjay Kadam Requirement of Vertical Handoff Mechanism in 4G Wireless Networks Ph. D Student,Computer Science Department, University of Pune, Pune 2011.

[7] W.Ma,Y.Fang, and P.Lin, "Mobility Management Strategy Based on User Mobility Patterns in Wireless Networks," IEEE Trans. Vehicular Technology, vol. 56, no. 1, pp. 322-330, Jan. 2007.

[8] Y.-B. Lin and A.-C. Pang, Wireless and Mobile All-IP Networks. John Wiley and Sons, 2005.

[9] D. Johnson, "Mobility Support in IPv6", IETF RFC 3775, 2004.

[10] R. Koodli, Ed., "Mobile IPv6 Fast Handovers", IETF RFC 5568, 2009

[11] H. Soliman, C. Castelluccia, K. E. Malki, L Bellier, "Hierarchical Mobile IPv6 (HMIPv6) Mobility Management”, IETF RFC 5380, 2008.

[12] S.Gundavelli, Ed., K. Leung, V. Devarapalli, K. Chowdhury, B. Patil, "Proxy Mobile IPv6", IETF RFC 5213, 2008.

[13] Qing-An Zeng \& Dharma P. Agrawal, (2002) "Handbook of Wireless Networks and Mobile Computing", John Wiley \& Sons Publishers.

[14] James Won-ki Hong \& Alberto Leon-Garcia, (2005) "Requirements for the Operations and Management of $4 \mathrm{G}$ networks", In Proc. of $19^{\text {th }}$ International Conference on Performance Challenges for Efficient Next Generation Networks, pp 981-990.

[15] http://www.tutorvista.com/-United States

[16] J. McNair \& F. Zhu, June (2004) "Vertical Handovers in Fourth-generation Multi-network Environments", IEEE Wireless Communications, Vol. 11, No. 3, pp 8-15.

[17] W. Chen \& Y. Shu, March (2005) "Active Application Oriented Vertical Handover in Next Generation Wireless Networks", In Proc. of IEEE WCNC'05, New Orleans, LA. 